\title{
Mechanical and Adhesion Properties of UV Curable Coating Based from Epoxidized Oil
}

\author{
Rahmah Mohamed,Wan Aimi Zalikha Ahmad Sidek and Ahmad Faiza Mohd \\ Faculty of Applied Sciences, Universiti Teknologi MARA, Shah Alam 40450, Selangor, Malaysia
}

Received: September 03, 2010 / Accepted: October 12, 2010 / Published: September 25, 2011.

\begin{abstract}
In this research, epoxidized oil (EO) has been exploited as a raw material in epoxy resin production. Epoxy resin produced from EO can be used for specific applications such as in coating industry. Conventional epoxy resin was said to be hazardous to human and is rather costly. On the other hand, EO which is sustainable from nature and cheaper, exhibits excellent properties such as adhesion to various substrates, good pigment wetting properties and thermally stable. The aim of this research is to substitute raw material of epoxy resin with epoxidized oil that exhibits similar properties as the conventional one. UV curable coating was prepared by copolymerization of EO with different monomers. Formulated resin was then applied on glass substrate and cured by ultraviolet (UV) radiation. From test performed, UV curable coating EO was found to possess excellent adhesion and scratch resistance. Coating samples have high adhesion force and passed high pencil grade. Hardness of this epoxidized system is high; all has minimum hardness of $5 \mathrm{~B}$ while it has low volatile organic compound (VOC) about $2 \%$ due to probably residual monomers. The system is solvent free and is an energy efficient coating. EO can be potential material for UV curable epoxy system suited to be applied for coating with different hardness and mechanical properties.
\end{abstract}

Key words: Epoxidized oil, epoxy resin, adhesion, scratch test, hardness.

\section{Introduction}

Epoxy resin was commercially introduced in late of 1930's. The resin is classified as thermosetting epoxide polymer which cured when mixed with a catalyzing agent. This kind of resin is produced mainly from the reaction between epichlorohydrin and bisphenol-A. By heating, epichlorohydrin and bisphenol-A undergo the ring opening mechanism which allow them to be converted into epoxy [1]. Epoxy resin possessed outstanding physical properties which include low cure shrinkage, no volatiles given off during cure, compatibility with a great number of materials, good adhesion, corrosion and chemical resistance and capability in electrical insulation.

Other than properties stated, epoxy resin system also capable to be cure in ambient or elevated

Corresponding author: Rahmah Mohamed, Ph.D., associate professor, research fields: polymer technology, polymer characterization, fabrication and materials, coating and green polymers. E-mail: greenkayangan@gmail.com, rahmahmd@salam.uitm.edu.my. temperatures with required only minimal pressure. It is widely used as adhesives and matrices due in good physical and chemical properties. In addition, this kind of resin can be applied and cured under various conditions such as outdoors. Due to its characteristics, epoxy resin has widely used in many applications especially in coatings industry.

Oil that regenerated via photosynthesis was synthesis thru epoxidation process with organic peracids. Oil after epoxidation or can be called epoxidized oil is mainly used as plasticizer, stabilizer, reactive modifier and diluents [2]. EO is low in cost and it also abundantly exist as well as biodegradability and sustainable. Hence, it has been exploited in this study to replace commercial raw materials of epoxy resin.

EO has good pigment wetting properties and flexibility combination with relatively low viscosity and low cost. It also exhibit specific properties such thermal stability, flexibility, resistance to chemical, 
biocompatibility, biodegradability, adhesion to metallic substrate, non electrical conductivity and non-flammability [3]. However, mechanical and adhesion properties of this type of coating needed to be study in order to ensure EO as a suitable material to produce resin for coatings and also to reduce cost in resin production.

Thermal and photochemical are common methods that have been used for crosslinking process of coatings on the surface of a substrate. Between these methods, UV curing (photochemical) appeared to be a more valuable technique than conventional thermal curing. UV curing tend to be a high speed process, low energy consumption because it can be carried out at room temperature and also environmentally friendly with nonvolatile evolution. Clear top coatings that used the UV curable systems may provide good surface protection for substrate such as metals, wood, paper, plastics and optical fibers [4].

Research by Ramya et al had utilized epoxidized oil as additional resin co-reactant added to the standard epoxy resins used in commercially bake system coating. The study had used up to $40 \%$ of epoxidized oil in its varied formulations. The effect of epoxidized oil on film properties were investigated by doing hardness test. Result of hardness test showed that film having low hardness with the association of replacement of rigid cycloaliphatic ring structure by flexible long chain fatty acids in the triglyceride. Use of epoxidized oil also reduces the cost of coating. Other than that, addition of other crosslinker had improved the film hardness but caused the reduction on impact resistance and flexibility [5].

Epoxidized oil was synthesized to toughen the tetrafunctional epoxy resin. $20 \%$ of epoxidized oil was blended with epoxy resins to modify the resin network. The modified blend resin was characterized by thermal and mechanical properties [6]. Based on the test on mechanical properties of resin, through work by Soo et al., addition of larger molecular weight of epoxidized oil into epoxy resins increased the flexibility properties of epoxy. Also, an increase in the content of epoxidized oil in epoxy resin had increased the toughness of blends [6].

\section{Experiment}

\subsection{Materials}

Acrylic acid was incorporated with EO in the oil modification process in order to produced acrylated epoxidized oil. Monomers to be copolymerized with acrylated epoxidized oil may comprise from copolymers with different ratios used.

\subsection{Modification of Oil}

Acrylated epoxidized oil (AEO) was obtained through oil modification process. EO and acrylic acid were filled into a reaction vessel equipped with thermometer, water condenser, mechanical stirrer and nitrogen inlet. The flask has been heat up until it reached temperature between $80-90{ }^{\circ} \mathrm{C}$ for about several hours. The crude product was filtered and washed for a few times after the reaction was completed [2].

\subsection{Preparation of Sample}

AEO were mixed with different monomers in various compositions. Mixture was then applied onto substrate by using Elcometer 4340 Automatic Film Applicator with $50 \mu \mathrm{m}$ in thickness. The UV curable coatings were irradiated using IST UV exposure machine. The dosage of UV radiation used is $8.5 \mathrm{~A}$ current with intensity of $0.107 \mathrm{~W} / \mathrm{cm}^{2}$ and $2 \mathrm{~m} / \mathrm{min}$ conveyer speed.ca (Table 1).

\subsection{Pencil Scratch Hardness Test}

Pencils of different grades were used in pencil hardness test. Test was carried out according to ASTM D 3363-74 whereby pencils were maintained at $45^{\circ}$ and pushed with uniform pressure onto the coated substrate. The surface of coating substrate was then examined for any superficial traces or destruction left by the pencil [4]. 
Table 1 Basic composition of materials contained in each coating mixture.

\begin{tabular}{llccll}
\hline Mixture & AEO (\%) & $\begin{array}{l}\text { Copolymer } \\
(\%)\end{array}$ & $\begin{array}{l}\text { A } \\
(\%)\end{array}$ & $\begin{array}{l}\text { Copolymer } \\
(\%\end{array}$ \\
\hline 50A & 50 & 0 & 50 & \\
10A40C & 50 & 10 & 40 & \\
20A30C & 50 & 20 & 30 & \\
30A20C & 50 & 30 & 20 & \\
40A10C & 50 & 40 & 10 & \\
50A & 50 & 50 & 0 & \\
\hline
\end{tabular}

\subsection{Pull off Test}

Adhesion strength between coating layer and substrate can be measured by doing pull off test. Test preparation included the step where a metal piece called dolly was glued onto the coating surface. The sample was left for overnight so, this will allow the resin between dolly and coating surface to be dried. The dolly was pulled off from sample using a device (Posi Test AT Pull-Off Adhesion Tester) that able to measure the force applied [7]. The steps were repeated three times for each samples and the average force applied for samples were taken.

\subsection{Volatile Organic Compound (VOC) Test}

Empty sample container was weighed. Container was filled with uncured AEO resin in the range of 3-5 g per container. Mass of container with resin was taken. Epoxy resin with various compositions was subjected into oven baked for certain hours using temperature of $120^{\circ} \mathrm{C}$. Mass of resin before and after drying was taken and percentage difference in mass over dry weight were calculated for VOC.

\subsection{Indentation Hardness Test}

Indentation hardness of coating samples was tested using Shimadzu's Dynamic Ultra Hardness Tester DUH-211S.

\section{Results and Discussion}

Table 2 showed the physical properties and the result of mechanical testing on UV curable coating samples. Apparently, all the coating samples appeared to be colourless and glossy on their physical appearances. The pencil hardness was termed as the softest pencil that left permanent induction on the surface of the coating [4]. During the test, all coatings had passed up to the $6 \mathrm{H}$ pencil hardness due to the observation that there is no mark left on the coatings. The result on coating sample had deviated when it was tested using pencil up to the $6 \mathrm{~B}$ pencil grade. Samples $50 \mathrm{C}, 10 \mathrm{~A} 40 \mathrm{C}$ and $20 \mathrm{~A} 30 \mathrm{C}$ only passed the pencil hardness test up to the $5 \mathrm{~B}$ pencil grade while the other coating samples, 30A20C, 40A10C and 50A does passed the test up to $6 \mathrm{~B}$ pencil grade. Thus, coating with higher amount of copolymer $\mathrm{A}$ in its mixture tend to have better pencil hardness compared to the coating with higher amount of copolymer $\mathrm{C}$.

\subsection{Adhesion}

From the Table 2, adhesion strength for all coatings was ranged between $0.9 \mathrm{Mpa}$ to $2.0 \mathrm{Mpa}$. The range $\mathrm{ab}$ is the force needed to pull out the coatings from the

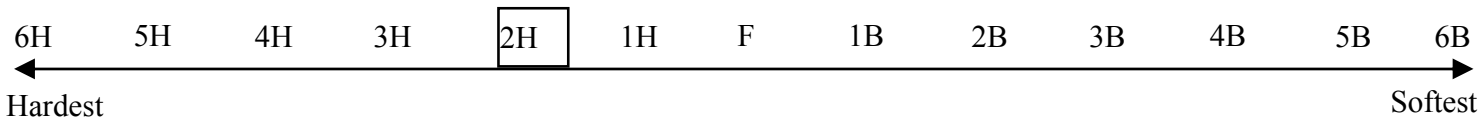

Fig. 1 Range of pencils used in testing from hardest to softest.

Table 2 Table of results for physical and mechanical properties of AEO resin.

\begin{tabular}{lllllll}
\hline Coating mixture & $50 \mathrm{C}$ & $10 \mathrm{~A} 40 \mathrm{C}$ & 20A30C & 30A20C & 40A10C & 50A \\
\hline Colour & Colourless & Colourless & Colourless & Colourless & Colourless & Colourless \\
Appearance & Glossy & Glossy & Glossy & Glossy & Glossy & Glossy \\
Pencil hardness & $5 \mathrm{~B}$ & $5 \mathrm{~B}$ & $5 \mathrm{~B}$ & $6 \mathrm{~B}$ & $6 \mathrm{~B}$ & $6 \mathrm{~B}$ \\
Adhesion $(\mathrm{MPa})$ & 0.96 & 0.99 & 1.02 & 1.12 & 1.63 & 1.95 \\
VOC $(\%)$ & 2.78 & 2.71 & 2.56 & 2.46 & 2.46 & 1.98 \\
\hline
\end{tabular}


glass substrate. Adhesion strength for samples was increased with the increasing in tetracrylate content in the coating mixture. This can be easily observed by the bar graph plotted in Fig. 2. In that bar graph, 50A with $50 \%$ of copolymer $\mathrm{A}$ in its mixture tend to have highest adhesion force compared to the non contained tetracrylate, 50C. Increase in adhesion force between samples may relate to the increase in surface energy of substrates. Based on the adhesion force possessed by each sample, it can be said that sample with higher content of copolymer A have higher surface energy compared to the sample with higher content of copolymer C. Surface energy of substrate have opposite relation with the contact angle of liquid coating mixture. High in surface energy of substrate tend to have lower contact angle of liquid coating mixture. The wetting of the substrate is high and this can lead to greater adhesion strength if it has been improved [8].

\subsection{Volatile Organic Compound (VOC)}

Based on Table 2 and Fig. 3, all coating samples have low VOC which is below 3\%. This showed that only small percentage of coating mixture had loss during the testing. Coating samples can be said to have low volatility and high reactivity which means that coating mixture can be cured in short period of time with the low emission of VOC. Journal reviewed by Giri [9] on "Genetic toxicology of epichlorohydrin" had reported that epichlorohydrin caused the respiratory and dermal toxicity in animals and humans. Also, the author had claimed that epichlorohydrin is carcinogenic towards humans and animals. Based on the VOC result, it can also discussed that the coating formulated based on EO does not give adverse effect on human health compared to the conventional epoxy resin. Low VOC showed that the coating is less toxicity and gives no harm to the environment.

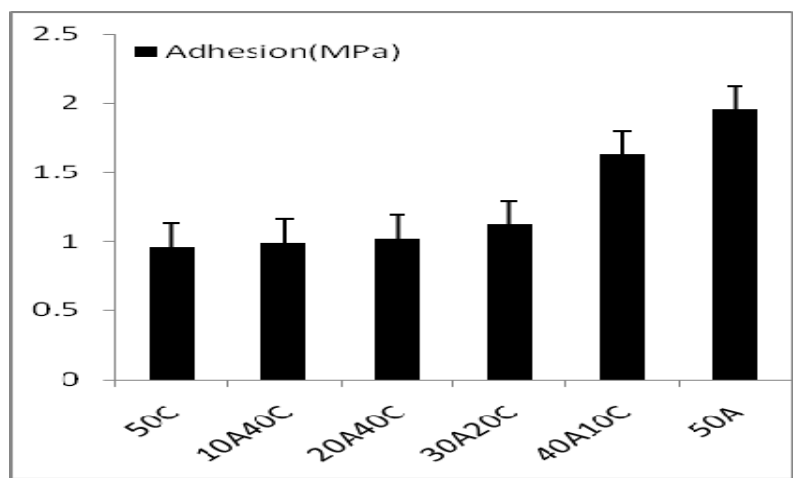

Fig. 2 Graph of adhesion strength for different AEO coating mixtures.

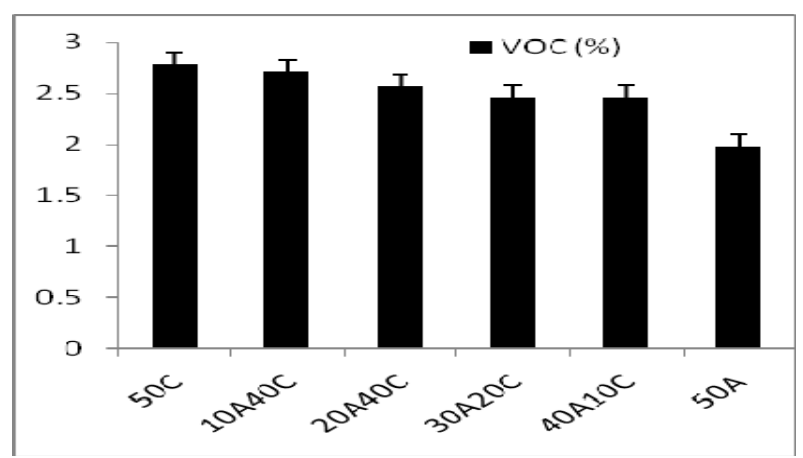

Fig. 3 Graph of VOC's percentage for different AEO coating mixtures.

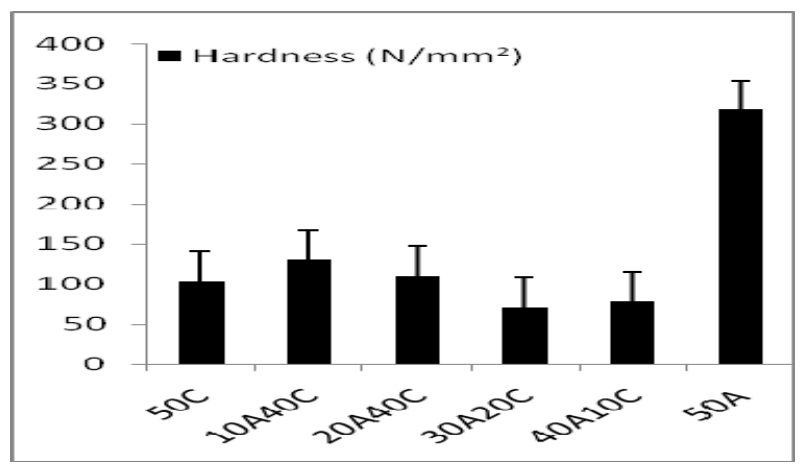

Fig. 4 Graph of indentation hardness for different AEO coating mixtures.

\subsection{Indentation Hardness}

Graph in Fig. 4 showed that 50A sample had obtained highest hardness value compared to the other samples which is about $320 \mathrm{~N} / \mathrm{mm}^{2}$. Sample that incorporated with $50 \%$ of copolymer $\mathrm{C}, 50 \mathrm{C}$ having approximately $50 \mathrm{~N} / \mathrm{mm}^{2}$ of hardness. Obviously, type of copolymer used in the sample gives effect to the hardness value. Use of copolymer A has resulted the 
hardness to be the highest while sample that incorporated with copolymer $\mathrm{C}$ tend to have lower hardness value. Sample with copolymer A possessed high crosslinking density which resulted in high hardness value. Also, hardness of coating system related to its tensile strength. High value of hardness tends to have high tensile strength [10].

Value of hardness reflects the concentration of porous particle as well as the presence of air bubble porosity [11]. 30A20C sample had showed the lowest hardness value $\left(\sim 70 \mathrm{~N} / \mathrm{mm}^{2}\right)$ among the other composition of coating system. This may due to the absorption of water or moisture of sample. It is learnt that absorbed water or moisture may act as plasticizer and thus reduced the hardness.

\section{Conclusions}

Based on the research done, EO has been successfully used as the substitution of common raw material of epoxy resin and thus exhibits similar properties as conventional epoxy resin. Testing done to evaluate coating performance had shown that all coating samples have good mechanical properties. All coating samples passed the high pencil grade in pencil hardness test. In fact, coating mixture with high copolymer A content has high pencil hardness. AEO mixed well with copolymers and adhere well to the substrate. Coating sample have good adhesion properties. Adhesion force of coating can reach up to 2 Mpa and high adhesion has high surface energy. Also, it can be concluded that coating is safe to use and environmentally friendly as it has low VOC and less emission. Incorporation of copolymer A exhibited high hardness value probably due to its high crosslinking density resulted from copolymer A.

\section{Acknowledgments}

The authors would like to express their highest gratitude to Ministry of Science, Technology and Innovation, Malaysia for funding this research.

\section{References}

[1] E.D. Walter, Plastic Chemistry and Technology, Texas Urethanes Incorporated, Austin, Texas, Litton Educational Publishing, Inc., 1979, p. 60.

[2] S.J. Park, F.L. Jin, J.R. Lee, Thermal and mechanical properties of tetrafunctional epoxy resin toughened with epoxidized soy bean oil, Material Science and Engineering A 374 (2004) 109-114.

[3] A.F. Mohd, Biopolymers from triglyceride oil as raw materials for coatings, in: Seminar on Current Development and Future Trend of Coatings Industry, Universiti Teknologi Mara, Shah Alam, Aug 20-21, 2008.

[4] V. Choudhary, N. Agarwal, I.K. Varma, Evaluation of bisacrylate terminated epoxy resins as coatings, Progress in Organic Coatings 57 (2006) 223-228.

[5] R. Ramya, S. Greg, B. Jamil, M. John, Cationic, thermally cured coatings using epoxidized soybean oil, Journal of Coatings Technology 72 (2000) 125-134.

[6] J.P Soo, L.J. Fan, R.L. Jae, Thermal and mechanical properties of tetrafunctional epoxy resin toughened with epoxidized soybean oil, Materials Science and Engineering 374 (2004) 109-114.

[7] J.R. Svendsen, G.M. Kontogeorgis, S. Kiil, C.E. Weinell, M. Grønlund, Adhesion between coating layers based on epoxy and silicone, Journal of Colloid and Interface Science 316 (2007) 678-68.

[8] M. Mohseni, Adhesion performance of an epoxy clear coat on aluminium alloy in the presence of vinyl and amino-silane primers, Progress in Organic Coatings 57 (2006) 307-313

[9] A.K. Giri, Genetic technology of epichlorohydrin: a review, Mutation Research 386 (1997) 25-38.

[10] D.K. Chattopadhyay, S.S. Panda, K.V.S.N. Raju, Thermal and mechanical properties of epoxy acrylate/methacrylates UV cured coatings, Progress in Organic Coatings 54 (2005) 10-19.

[11] L. Ehrnford, Composite resins with a condensable inorganic phase, J. Dent. Res. 60 (1981) 1759-1766. 